\title{
Mejorar la convivencia escolar a través de la lectura mediante la investigacion como estrategia pedagogica ${ }^{1}$
}

\section{Improve school coexistence through reading through research as a pedagogical strategy}

DOI: http://dx.doi.org/10.17981/cultedusoc.9.3.2018.65

Artículo de investigación. Fecha de recepción: 15/06/2018. Fecha de aceptación: 27/11/2018

Georgina Robayo-Puentes²,

Dairo Gil-Rodríguez; Dionisia Guillén-Villamil; Margoth Vizcaíno-Camacho;

Sandy Manjarrés-Bolaño; Sixta Londoño-Barraza y Vialnellys Barrios-Acosta ${ }^{3}$

Institución educativa tercera mixta, sede centro educativo José Antonio Galán (Colombia) geropu1403@outlook.com

Para citar este artículo:

Gil-Rodríguez, D., Guillén-Villamil, D., Vizcaíno-Camacho, M., Manjarrés-Bolaño, S., Londoño-Barraza, S. y Barrios-Acosta, V. (2018). Mejorar la convivencia escolar a través de la lectura mediante la investigacion como estrategia pedagogica. Cultura. Educación y Sociedad 9(3), 543-550. DOI: http://dx.doi.org/10.17981/cultedusoc.9.3.2018.65

\section{Resumen}

La mala convivencia que se vive en las instituciones educativas se ha convertido en un factor perturbador de la convivencia escolar; se considera que es necesario intervenir para lograr un cambio significativo dentro de las aulas de clase. El presente artículo tiene como objetivo mejorar la convivencia escolar utilizando la lectura mediante la investigación como estrategia pedagógica (IEP); Se realizó un estudio de tipo cualitativo bajo el modelo de investigación descriptivo, se utilizó una población conformada por treinta y ocho (38) estudiantes de cuarto (4) grado de primaria de la institución educativa Tercera Mixta, sede José Antonio Galán. Durante el proceso investigativo, se aplicaron técnicas e instrumentos tales como la observación directa, diarios de campo; La investigación logro captar la atención de los estudiantes reduciendo la mala convivencia dentro y fuera del aula de clase y aumentando el aprovechamiento del tiempo libre utilizando como estrategia la lectura fortaleciendo la investigación.

Palabras clave: convivencia escolar, lectura, investigación como estrategia pedagógica (IEP)

\section{Abstract}

The bad coexistence that lives in the educational institutions has become a disturbing factor of the school coexistence; It is considered that it is necessary to intervene to achieve a significant change within the classrooms. The objective of this article is to improve school coexistence by using reading as a pedagogical strategy (IEP); A qualitative study was carried out under the model of descriptive research, a population made up of thirty-eight (38) students of fourth (4) grade of primary school of the Third Mixed educational institution, José Antonio Galán. During the investigative process, techniques and instruments were applied such as direct observation, field diaries; The research managed to capture the attention of the students by reducing the bad coexistence inside and outside the classroom and increasing the use of free time using reading as a strategy to strengthen research.

Keywords: school coexistence, reading, research as a pedagogical strategy (IEP)

\footnotetext{
1 Este artículo ha sido derivado del Programa de Fortalecimiento de la Cultura Ciudadana y Democrática CT+I a través de la IEP apoyada en TIC en el Departamento de Magdalena: CICLON

2 Líder del grupo de investigación "Unidos en el Fortalecimiento de la Cultura y La Convivencia Escolar".

3 Docentes de la institución educativa tercera mixta, sede centro educativo José Antonio Galán, del grupo de investigación "Unidos en el Fortalecimiento de la Cultura y La Convivencia Escolar".
}

- The author; licensee Universidad de la Costa - CUC. 


\section{Introducción}

La violencia y el mal trato que se vive a diario en las aulas de clases han pasado a hacer parte del hábito común entre estudiantes y docentes. Ha dejado de ser motivo de asombro o sorpresa, y esto solo lleva a que esté hábito más que promoverse se pueda perpetuar en el diario escolar. El tema de violencia escolar simplemente hoy en día no tiene espacio en la agenda de investigación educativa nacional (Gómez, Nashiki 2005).

Por otro lado, de manera correctiva, existen unas instituciones preocupadas por desarrollar estrategias adecuadas para poder enfrentar situaciones de indisciplina y/o violencia, también se ha informado de investigaciones que se realizan con la finalidad de abarcar temas como el comportamiento de los estudiantes en el aula de clases, incluyendo faltas de respeto al maestro, el maltrato, el acoso y el abuso entre compañeros o entre profesores y alumnos, bajo el nombre de violencia, con el propósito de ir planteando estrategias para mejorar la disciplina (Furlan, 2005).

En países como España, hacia inicio de la década de los noventa, se empezaron a desarrollar y a diseñar estrategias e iniciativas que iban encaminadas a hacer frente a la problemática hoy en día resaltada de la violencia escolar. Distintas propuestas innovadoras centran sus esfuerzos en superar la conflictividad y aumentar la sana convivencia dentro y fuera de las aulas de clases (Ruiz, Félix, Alamillo, 2009). El fomentar la lectura en los estudiantes para ocupar y entretener sus mentes durante los espacios libres se considera una muy buena estrategia pedagógica para hacer crecer en el pensamiento de los estudiantes ideas productivas que en consecuencia distraigan al alumno de cosas vacías que puedan afectar sus relaciones interpersonales.

Los docentes, directivos y coordinadores de instituciones que presentan problemas de convivencia entre sus alumnos consideran el clima escolar bastante deteriorado, pues se entiende como clima escolar al resultado de las relaciones interpersonales que tienen lugar en la escuela, mientras que los alumnos y alumnas lo vinculan exclusivamente a los contextos de aprendizaje (Murillo, Becerra, 2009). El clima social de una institución educativa se concreta en las relaciones sociales y las diferentes percepciones de los agentes de la comunidad educativa, al llevar acabo procesos de formación y tareas innovadoras de enseñanza- aprendizaje (Rivilla, González, 2010).

Al momento de hablar de violencia esta debe verse desde dos puntos de vista que guardan relación entre sí, están son la violencia directa y la indirecta, donde la primera hace referencia a las situaciones cara a cara, donde se utiliza la fuerza física o la palabra para resolver diferencias, y la indirecta se ve manifestada a través de injusticias sociales, la carencia de servicios médicos y asistenciales, entre otras. Entendiendo como violencia al impedimento del desarrollo de potencialidades humanas y privación de los derechos (Orellano, 2007).

Aprender a vivir consigo mismo en ocasiones resulta ser un poco complicado, ahora pensar en convivir con los demás, con la naturaleza y con las culturas seria uno de los grandes retos de la sociedad actual. En la educación del siglo XXI este es uno de los objetivos a los que más interés se les ha puesto, por ende se han venido desarrollando estrategias que ayuden a incrementar los aprendizajes básicos de una convivencia social. En el caso de las instituciones educativas este es un reto que incluye tanto a docentes como estudiantes y padres de familia, pues es compromiso de todo el personal involucrado el buscar soluciones para dicha problemática (Leiva, Paz, 2009). A nivel mundial, los primeros estudios sobre violencia en las escuelas se realizaron en Noruega y Suecia, en las décadas de 1960 y 1980, los hechos de violencia impiden de 
cierta forma que los estudiantes desean asistir a las escuelas y pierdan concentración en clases, lo que lleva a que el problema empeore (Mesa, castillo, Sotos, Roa, 2013).

\section{Convivencia escolar}

La convivencia escolar tiene mucha relación e influye en uno de los temas básicos de la pedagogía: el aprendizaje, es decir el proceso mediante el cual un sujeto adquiere el conocimiento para desarrollar diversas actividades y comprender nuevos significados (Ianni, 2003). Debido a esto constituye uno de los aspectos más importantes en las relaciones humanas, pues de la educación y el buen comportamiento hablan por sí solas de quien eres, debido a que como manifestación natural de estas relaciones surgen desacuerdos, comunicación inadecuada, etc, que llevan a conflictos interpersonales (Grande, 2010).

No podemos evitar convivir con otras personas, la relación con los demás es inevitable en cualquier sociedad, desde que nacemos comenzamos conviviendo con aquellas personas que hacen parte de nuestro núcleo familiar y a medida que vamos creciendo nos vamos integrando a otros grupos que despliegan actividades en escenarios diferentes, uno de los espacios más importantes y del cual no podemos escapar es la escuela, el lugar a donde asistimos desde muy pequeños para recibir educación y en el cual nos encontramos con muchas personas más, cada una con diferente forma de pensar y actuar (Ramírez, Justicia, 2006).

\section{La escuela como espacio propicio para actos de violencia}

El aumento de la complejidad que adquieren las escuelas, hace de estas un lugar complicado para quienes a estas acuden, por ende la convivencia dentro de las aulas de clases se torna un poco compleja.
Existen muchos elementos y muchas variables, que hacen de la escuela un lugar propicio de actos de violencia y que de cierta forma impiden que la escuela cumpla eficazmente con las funciones que se les ha encomendado. (Ramírez, Justicia, 2006). Algunos de los aspectos que influyen en el aumento de la mala convivencia en las escuelas son:

- En ocasiones las conductas agresivas que se presentan entre estudiantes pasan por desapercibidas para los docentes, esto debido a la gran cantidad de estudiantes que acuden a las escuelas.

- Algunos padres de familia toman actos de violencia como actitudes o compartimientos normales entre niños.

- El silencio de los estudiantes agredidos, por miedo a recibir peores tratos por parte de sus agresores, con quienes a diario deben convivir

- Los estudiantes conviven diariamente durante varias horas al día, lo que hace aumentar las diferencias y desacuerdos entre ellos (Cid, Díaz, Pérez, Torruella y Valderrama, 2008).

- La lectura como estrategia pedagógica

- La lectura se realiza como estrategia pedagógica y para que se convierta en una herramienta didáctica donde se manifieste la lúdica y la participación para generar aprendizajes significativos, donde los docentes se sientan satisfechos en el desarrollo de las actividades dentro y fuera del aula de clases (Riascos, 2014).

- El interés por analizar géneros narrativos, se basa en el hecho de que los relatos no son solo un medio para comunicar experiencias, sino que también reflejan la forma en que comprendemos el mundo, la vida y a nosotros mismos. En este sentido, la lectura de cuentos ayudara a la conformación de un formato narrativo que permita al niño organizar la información de forma coherente (Borzone, 2005). 
- La lectura y su nivel de interpretación se hace más compleja a medida que se van superando los diferentes niveles en la escuela, la lectura dialógica es el proceso de leer y comprender un texto en el que personas expresan de manera profunda sus interpretaciones y reflexionan críticamente sobre el mismo y su contexto (Valls, Soler y Flecha, 2008).

\section{La lectura mediante la}

IEP apoyada en TIC

- El hacer parte de los currículos de la IEP apoyados en TIC, hoy en día, se convierte en el principal reto para cada una de las personas interesadas en el proceso de formación de los estudiantes. La IEP a través de la utilización de las TIC, propende por generar cambios significativos en los procesos de formación escolar, y que cada proceso que se desarrolle en la institución educativa, este ligada a procesos de investigación, por ello resulta adecuado decir que la investigación como estrategia pedagógica ejerce un impacto vinculante entre la práctica pedagógica y la investigación, se le brinda a las niñas la posibilidad de apropiarse de la lógica del conocimiento y de herramientas propias de la ciencia con la mirada puesta en la sociedad y sus necesidades, proyectándonos al conocimiento significativo al que se refiere Ausubel (2001).

- En cuanto a las cifras de violencia escolar, el municipio de Fundación no cuenta con el registro estadísticos de los casos que se presentan en cada una de las instituciones educativas ya sean públicas o privadas, esto favorecido por la ausencia del comité municipal de convivencia escolar, por esto el manejo de las agresiones carece de un dato estadístico confiable. Ante esto se hace necesario el implementar estrategias encaminadas a superar las situaciones de agresividad en las instituciones, para ello es pertinente que los estudiantes utilicen la lectura mediada por la IEP y apoyada en las TIC, en el manejo y resolución de conflictos de manera pacífica, utilizando el dialogo como medio indispensable para resolver las diferencias y así promover espacios de sana convivencia en la institución. Esta es una de las metas del proyecto y para ello se utilizará el área de Lengua Castellana, en esta área un aprendizaje básico es la lectura, no sólo saber leer, sino comprender, asimilar el mensaje del texto leído, por lo tanto se convierte en una oportunidad para fortalecer las relaciones interpersonales y la Investigación como Estrategia Pedagógica permite generar espacios en donde el estudiante se apropie de la problemática del conflicto y explore en la búsqueda de sus raíces, de esta manera puede generar cuestionamientos en caminados a cambiar las conductas agresivas por otras en las que medie el dialogo.

\section{Actitudes agresivas de los estudiantes en el aula de clases}

No existe ninguna duda de que el éxito del profesor en el manejo de la clase se basa en dos importantes aspectos: la autoridad y la destreza. Por lo general si el profesor posee autoridad también se caracteriza por su destreza o viceversa. La principal fuente de recursos del profesor, la más provechosa, está en él mismo, en su propia personalidad (Alcázar, 2003).

El comportamiento de los estudiantes en el aula de clases varía según el medio en el que se desarrollan, ya que su conducta depende de los valores y reglas aprendidas en el hogar. Los momentos que el docente comparte con el niño en el aula de clases, ayuda al maestro a percibir conductas anormales, familiarizarse con ellas y tomar medidas a tiempo. La conducta del 
niño permite conocer su nivel de satisfacción o frustración en la escuela, si este, no se siente satisfecho con lo recibido en la escuela, puede llegar a presentar conductas agresivas con sus compañeros e incluso con los docentes. Las conductas agresivas son aquellas actitudes y respuestas violentas que ocasionan daño a uno mismo y al medio que nos rodea. La agresividad la podemos clasificar de diversas maneras: física, moral, verbal, psicológica, material, ofensiva o de intimidad, estas son las diferentes formas de como puede surgir la agresividad (Capa, Onela, 2017).

\section{Metodología}

\section{Diseño}

Se realizó una investigación cualitativa, de estudio con diseño descriptivo están encaminados a especificar las características, perfiles y propiedades de personas, grupos, comunidades, procesos, objetos o cualquier otro fenómeno que sea sometido a un análisis. (Hernández, Fernández \& Baptista, 2010).

Como lo señala Arias (2012), los estudios descriptivos miden de forma independiente las variables y aun cuando no se formulen hipótesis, tales variables se encuentran formuladas en los objetivos de la investigación; de esta manera generar información, procurando describir situaciones, eventos y hechos que se vinculan con las variables objeto de estudio para fomentar la formación en valores mediante la investigación como estrategia pedagógica (IEP) en educación básica. Partiendo de lo señalado por los autores antes citados, la investigación descriptiva está orientada concretamente a que el investigador logre describir su objeto de estudio, empleando recursos apropiados y realizando el procedimiento adecuado, que permita obtener del fenómeno estudiado información adecuada para el establecimiento de un juicio propio cuando esté realizando dicha descripción.

\section{Participantes}

La población beneficiaria con este estudio corresponde a la comunidad estudiantil de la sede José Antonio Galán, anexa de la Institución Educativa Departamental Tercera Mixta, del municipio de Fundación Magdalena. Se seleccionaron treinta y ocho (38) estudiantes de cuarto (4) grado de primaria jornada de la tarde, cuyas edades oscilan entre nueve (9) a diez (10) años, que presentan situaciones adversas de convivencia. Con ellos se llevaron a cabo actividades que iban relacionadas directamente con la mejora de su convivencia y desarrollo.

\section{Técnicas e instrumentos}

Se utilizaron técnicas propias de la investigación cualitativa, como observación directa al participante y diario de campo, las cuales permitieron poner en evidencia el entorno y el espacio donde los estudiantes se desenvuelven dentro y fuera del aula de clases, permitiendo así sistematizar las experiencias aportando estrategias adecuadas para lograr una mejora notable del comportamiento de los estudiantes en el periodo escolar.

\section{Procedimiento}

El proceso de investigación se realizó basándose en cinco (5) momentos las cuales detallan lo planteado y ejecutado durante la investigación de la estrategia propuesta para mejorar la convivencia de los estudiantes.

- Momento (1): Convocatoria y acompañamiento para la conformación de los grupos de investigación; en este primer momento se toma los participantes y se genera la pregunta problematiza dora de la investigación.

- Momento (2): Identificación y definición de la temática de investigación, se realiza la indagación teórica en la investigación. 
- Momento (3): Diseño de las trayectorias de indagación, se seleccionaron en consenso los métodos, recursos y estrategias de la investigación.

- Momento (4): Producción de saber y conocimiento, aquí se realizó la sistematización de toda la investigación.

- Momento (5): Apropiación social del conocimiento, se divulgo el proceso de investigación y resultados obtenidos, en unos espacios de apropiación municipal "El maestro tiene la palabra".

\section{Resultados}

Esta investigación pretende dar a entender la importancia que tiene el emplear la lectura mediante la IEP para fortalecer y mejorar la convivencia en los estudiantes. $\mathrm{Al}$ ser la lectura un proceso en el cual se aumenta y se estimula el intelecto de quienes la aprovechan de manera positiva, el individuo apropia un conocimiento externo y diferente al ya conocido, aumentando su capacidad de pensamiento en medio de las actividades realizadas dentro y fuera de las aulas en sus diferentes dimensiones, pudiendo así adquirir hábitos y desarrollar formas de pensamiento que lo lleven a mejorar la forma de sobrellevar las diferentes situaciones presentadas a su alrededor.
Como se mencionó anteriormente en la investigación, se utilizaron técnicas e instrumentos tales como la observación directa y diarios de campo; logrando que los participantes y cuerpo docente identificaran la problemática dentro de las aulas de clase. Utilizando estrategias pedagógicas para fortalecer la convivencia escolar como la lectura mediante la investigación como estrategia pedagógica se pudieron identificar algunos de los factores que incidían en el mal comportamiento de los estudiantes en el aula de clases.

1. La falta de participación de los estudiantes en los grupos de investigación, y la falta de realización de actividades lúdicas que de cierta forma llamen la atención a los estudiantes y los inviten a participar.

2. Falta de atención por parte de los padres de familia y docentes durante los espacios libres de los niños.

3. Desactualización en el uso de herramientas del conocimiento e información actuales.

4. El miedo de los estudiantes por expresar lo que sienten durante el desarrollo de las diferentes actividades.

5. Falta de atención y acatamiento de normas.

TABLA 1

Actividades realizadas para mejorar la convivencia

\begin{tabular}{|c|c|c|}
\hline Actividad & Participantes & Resultados \\
\hline $\begin{array}{l}\text { Reuniones de los directivos } \\
\text { con los padres de los } \\
\text { estudiantes involucrados. }\end{array}$ & $\begin{array}{l}\text { Directivos y padres } \\
\text { de familia. }\end{array}$ & $\begin{array}{l}\text { Propuestas, acuerdos y soluciones para } \\
\text { manejar de manera adecuada la situación } \\
\text { presentada en los estudiantes. }\end{array}$ \\
\hline $\begin{array}{l}\text { Organización de mesas } \\
\text { redondas con los estudiantes } \\
\text { para conocer sus diferentes } \\
\text { puntos de vista sobre lo vivido } \\
\text { a diario en la institución. }\end{array}$ & $\begin{array}{l}\text { Docentes y } \\
\text { estudiantes. }\end{array}$ & $\begin{array}{l}\text { Ideas acerca de los posibles obstáculos que } \\
\text { se presentan en los estudiantes, y que hacen } \\
\text { que estos no actúen de la mejor manera ante } \\
\text { las diferentes situaciones presentadas en la } \\
\text { escuela. }\end{array}$ \\
\hline $\begin{array}{l}\text { Jornadas de lectura con los } \\
\text { estudiantes. }\end{array}$ & $\begin{array}{l}\text { Docentes y } \\
\text { estudiantes. }\end{array}$ & Motivación y ampliación del conocimiento. \\
\hline $\begin{array}{l}\text { Espacios de actividades } \\
\text { lúdicas fuera de clase. }\end{array}$ & $\begin{array}{l}\text { Estudiantes, padres } \\
\text { de familia y docentes. }\end{array}$ & $\begin{array}{l}\text { Mayor interacción entre los estudiantes, e } \\
\text { identificación de diferencias entre ellos. }\end{array}$ \\
\hline
\end{tabular}

Fuente: elaboración propia. 
Dentro del plan de acción se implementaron las siguientes actividades para la implementación del proyecto de investigación (tabla 1).

Al conocer los diferentes criterios que de cierta forma inciden en el desarrollo de la buena convivencia de los niños en las escuelas, se hizo necesaria la elaboración de las diferentes actividades que colaborarían en la solución del problema planteado. Obteniendo como resultado una mejora notoria en el desarrollo y convivencia de los niños durante sus jornadas de estudio. Los estudiantes ahora ocupan sus espacios libres en la elaboración de proyectos de investigación y en la realización de lecturas de niveles un poco más avanzados, lo que ocupa su mente y distrae de actividades que puedan perturbar la convivencia y tranquilidad de los niños.

\section{Conclusiones}

En esta investigación y partiendo de los resultados obtenidos, se pudo observar y dar cuenta de la necesidad de desarrollo del mismo, puesto que urge la mejora de la convivencia y el comportamiento de los estudiantes frentes a las diferentes situaciones y experiencias vividas en el aula de clases a través de la lectura. Las diferentes problemáticas que han venido presentando los estudiantes en temas de convivencia están muy relacionadas con la falta de aprovechamiento de sus horas libres, puesto que prefieren dedicarse a actividades sin ningún tipo de enseñanza formativa, dejando a un lado sus compromisos personales e institucionales.

Utilizar la lectura mediante la IEP, es una de las estrategias propuestas como solución al problema de la convivencia actualmente presentada en las instituciones, por esto, en el presente proyecto se propuso el uso de esta herramienta como recurso para atender la problemática previamente planteada. De cierta forma los estudiantes ven la lectura como una forma de entretenimiento. siempre y cuando se trate de temas que captan su atención, empezar por eso podría ayudar a desarrollar nuevos pensamientos e incluso a mejoran su actitud. La elaboración del proyecto permitió reconocer la importancia de la lectura como herramienta pedagógica para fortalecer el aprendizaje en los niños, niñas y jóvenes. Durante el proceso investigativo, se aplicaron técnicas e instrumentos tales como la observación directa, y diarios de campo, siendo estas las técnicas más adecuadas cuando se trata de un estudio de tipo cualitativo, logrando así que la comunidad educativa pueda identificar la problemática presente en la institución.

Se intentó desarrollar en el Centro Educativo en un lugar en el que se pueda convivir de manera sana y adecuada, presentando a los estudiantes las diferentes estrategias que les ayudarían a mejorar los problemas de convivencia, para que estas puedan ser aprendidas y aplicadas dentro y fuera del aula de clases. Una vez logrado esto poder gozar de la armonía y expresar con mucho orgullo y felicidad que una de las problemáticas más graves y más presentadas en las instituciones, gracias a un buen manejo estratégico pude ser solucionado. Todo esto conllevaría al buen prestigio de la institución y a la posibilidad de egresar estudiantes con una formación digna y adecuada.

\section{Referencias}

Alcazar, J. (2000). Convivencia y Disciplina Escolar: El Gobierno de la Clase. Recuperado de http://fresno.pntic.mec. es/ emod0002/disciplina.htm

Arias, F. (2006). El Proyecto de Investigacion. Introduccion a la Metodologia Cientifica. Caracas: Episteme.

Ausubel, D. (2002). Adquisicion Y Retencion del Conocimiento: Una Perspectiva Cognitiva. Barcelona: Paidos Iberica. 
Borzone, A. (2005). La Lectura de Cuentos en el Jardín Infantil: Un Medio para el desarrollo de Estrategias cognitivas y lingüísticas. (2005). Scielo, 14(1). 192209. http://dx.doi.org/10.4067/S071822282005000100015

Caballero, G. (2010). Convivencia Escolar. Un Estudio sobre Buenas Prácticas. Dialnet, 3(1). 154-170.

Furlan, A. (2006). Problemas de Indiciplina y Violencia en la Escuela. Revista Mexicana de Investigacion Educativa, 10(26). 631-639.

Gomez, N. (2005). Violencia e Institución Educativa. Revista Mexicana de Investigacion Educativa, 10(26). 693-718.

Hernandez R. (2010). Metodologia De La Investigacion. Mexico D.F.: Mcgraw Hil.

Ianni, N. (2003). La Convivencia Escolar: Una tarea necesaria, posible y compleja. Oei, 2(1). 1-11.

Marquez, C. (2017). Plan de intervención cognitivo conductual para modificar conductas agresivas en Niños y Niñas de 7 Años. Machala: Universidad Tecnica de Machala.

Medina, A. y Cacheiro, M. (2010). La Prevención de la Violencia: La implicación de la Comunidad educativa para evitar situaciones de acoso escolar. Revista de Pedagogia, 62(1). 93-107.
Murillo, P. (2009). Las percepciones del clima escolar por directivos, docentes y alumnado mediante el empleo de redes semánticas naturales. Su importancia en la gestión de los centros educativos. Revista De Educacion, 350(1). 375-399.

Ortega, R. (2009). Protagonismo de la atención a la convivencia en la prevención de la conflictividad y la violencia escolar. Informacion Psicologica, 95(1). 4-14.

Palomino, L. (2009). Los problemas de convivencia escolar: percepciones, factores y abordajes en el aula. Revista de Investigaciones Unad, 8(2). 199-221.

Cid, P., Díaz, A., Pérez, M., Torruella, M. y Valderrama, M. (2008). Agresión y violencia en la escuela como factor de riesgo del aprendizaje escolar. Scielo, XIV(2). 21-30. http://dx.doi.org/10.4067/ S0717-95532008000200004

Valls, R. Soler, M. y Flecha, R. (2008). Lectura Dialógica: Interacciones que mejoran y aceleran la lectura. Revista iberoamericana de educación, 46(1). 7187.

Valverde, Y. (2014). Lectura y Escritura con Sentido y Significado. Como Estrategia De Pedagógica En La Formación De Maestros. Fedumar Pedagogia Y Educacion, 1(1). 71-10. 